\title{
Dabigatran bleed risk with closed head injuries: are we prepared?
}

\author{
Clinical article
}

\author{
Michael W. Parra, M.D., ${ }^{1,2}$ Lloyd Zucker, M.D., ${ }^{1}$ Eric S. Johnson, D.O., ${ }^{1,2}$ \\ Diane Gullett, R.N., B.S.N., M.P.H., ${ }^{1}$ Cristina Avila, B.S., ${ }^{1,2}$ \\ Zachary A. Wichner, D.O., M.P.H., ${ }^{1,2}$ and Candace R. KokARam, M.S.N. ${ }^{1}$
}

${ }^{1}$ Delray Medical Center/Provisional Level I Trauma Center, Delray Beach; and ${ }^{2}$ Nova Southeastern

University College of Osteopathic Medicine, Fort Lauderdale-Davie, Florida

Object. The direct thrombin inhibitor dabigatran has recently been approved in the US as an alternative to warfarin. The lack of guidelines, protocols, and an established specific antidote to reverse the anticoagulation effect of dabigatran potentially increases the rates of morbidity and mortality in patients with closed head injury (CHI). Confronted with this new problem, the authors reviewed their initial clinical experience.

Methods. The authors retrospectively reviewed all cases of adult patients (age $\geq 18$ years) who sustained CHI secondary to ground-level falls and who presented to the authors' provisional regional Level I trauma center between February 2011 and May 2011. The authors divided these patients into 3 groups based on anticoagulant therapy: dabigatran, warfarin, and no anticoagulants.

Results. Between February 2011 and May 2011, CHIs from ground-level falls were sustained by 5 patients while on dabigatran, by 15 patients on warfarin, and by 25 patients who were not on anticoagulants. The treatment of the patients on dabigatran at the authors' institution had great diversity. Repeat CT scans obtained during reversal showed 4 of 5 patients with new or expanded hemorrhages in the dabigatran group, whereas the warfarin group had 3 of 15 $(\mathrm{p}=0.03)$. The overall mortality rate for patients sustaining $\mathrm{CHI}$ on dabigatran was $2(40 \%)$ of 5 , whereas that of the warfarin group was $0(0 \%)$ of $15(\mathrm{p}=0.05)$.

Conclusions. It is critical for physicians involved in the care of patients with $\mathrm{CHI}$ on dabigatran to be aware of an elevated mortality rate if no treatment protocol or guideline is in place. The authors will soon implement a reversal management protocol for patients with $\mathrm{CHI}$ on dabigatran at their institution in an attempt to improve efficacy and safety in their treatment approach.

(http://thejns.org/doi/abs/10.3171/2013.3.JNS12503)

\section{Key Words • dabigatran • warfarin • closed head injury • anticoagulation reversal protocol $\quad$ traumatic brain injury}

$\mathrm{T}$ HE direct thrombin inhibitor dabigatran has recently been approved in the US as an alternative to warfarin for risk reduction in stroke and systemic embolism in patients with nonvalvular atrial fibrillation. Several studies assessing the risk of major bleeding complications have shown dabigatran to have a reduced amount of risk compared with other anticoagulants..$^{12,14,18,21}$ However, the lack of guidelines, protocols,

\footnotetext{
Abbreviations used in this paper: $\mathrm{CHI}=$ closed head injury; FFP = fresh frozen plasma; GCS = Glasgow Coma Scale; $\mathrm{ICH}=$ intracranial hemorrhage; IPH = intraparenchymal hemorrhage; ISS = Injury Severity Score; NAC = no anticoagulants; $\mathrm{PCC}=$ prothrombin complex concentrate; $r F V I I a=$ recombinant human coagulation factor VIIa; $\mathrm{SAH}=$ subarachnoid hemorrhage; $\mathrm{SDH}=$ subdural hemorrhage.
}

and an established specific antidote to reverse the anticoagulation effect of dabigatran potentially increases morbidity and mortality in patients with CHI. Confronted with this new problem, we reviewed our initial clinical experience.

\section{Methods}

\section{Patient Population}

We retrospectively reviewed all adult patients (age $\geq$ 18 years) with CHI secondary to ground-level falls presenting to our provisional regional Level I trauma center

This article contains some figures that are displayed in color online but in black-and-white in the print edition. 
between February 2011 and May 2011. Ground-level falls were placed into 2 separate categories: 1) falls from stairs or steps, sidewalk or curb, and escalator; and 2) falls from a standing or seated position. We divided these patients into 3 groups based on their anticoagulant therapy: dabigatran, warfarin, and NAC. Patient characteristics, comorbidities, mechanism of injury, ISS, GCS scores at admission and discharge, laboratory data, CT scan findings, results of neurological examinations, treatment modality variables, and survival status were obtained from our trauma registry.

All patients with $\mathrm{CHI}$ who were on warfarin were placed on a standardized reversal protocol similar to the revised protocol used by Ivascu et al. ${ }^{6}$ Patients with evidence of an ICH on CT scans received an immediate transfusion of $2 \mathrm{U}$ of type AB FFP, followed by another transfusion of $2 \mathrm{U}$ of type-specific FFP. These patients also received vitamin $\mathrm{K}$ intravenous piggyback and an immediate neurosurgery evaluation. Due to the lack of an established protocol, there were variations in the treatment methods for the patients with $\mathrm{CHI}$ who were on dabigatran. The radiologist on call, who was blinded to both clinical findings and the patient's anticoagulant medications, interpreted the CT scan findings. All suspected hypertensive ICHs and nontraumatic SAHs were excluded from our study.

\section{Statistical Analysis}

Statistical analysis was conducted using IBM SPSS Version 20. The $\mathrm{p}$ values for categorical variables (sex, CT findings, associated medications, and deaths) were derived from the Fisher exact test, and for continuous variables (age, ISS, and GCS score) an independent-sample t-test was used. All $\mathrm{p}$ values were 2-tailed and values $<0.05$ were considered to indicate statistical significance.

\section{Results}

Between February 2011 and May 2011, CHIs from ground-level falls were sustained by 5 patients while on dabigatran, by 15 patients on warfarin, and by 25 who were not on anticoagulants. Of the 5 patients on dabigatran, 4 fell from a standing and 1 fell from a sitting position. Of the 15 patients on warfarin, 14 fell from a standing position and 1 fell from stairs. Of the 25 patients who were not receiving anticoagulants, 24 fell from a standing position and 1 fell from a skateboard. Of the 5 patients in the dabigatran group, the mean age was 82 years (range 79-85 years), and the male/female ratio was $3: 2$. The mean age of the 15 patients in the warfarin group was 84 years (range $71-95$ years), with a male/female ratio of 2:1. The 25 patients in the NAC group had a mean age of 75 years (range 19-93 years), with a male/female ratio of 1:1. The mean ISS score was 20 (range 4-25) in the dabigatran group, 16.5 (range 4-25) in the warfarin group, and 13.6 (range 2-30) in the NAC group. The dabigatran group had a mean GCS score of 14.6 at presentation, in the warfarin group the GCS was 14.6, and in the NAC group it was 14.2. Excluding deaths, the mean GCS scores at discharge were 14.3 in the dabigatran group, 14.5 in the warfarin group, and 14.8 in the NAC group.
The most common finding on CT scans in the dabigatran group was SAH (4 of 5), SDH (1 of 5), and IPH (2 of 5). The most common findings in the warfarin group were SDH (12 of 15), IPH (5 of 15), and SAH (3 of 15). The most common findings on CT scans in the NAC group were SDH (12 of 25), SAH (11 of 25), and IPH (3 of 25). Several patients underwent surgeries related to their $\mathrm{CHI}$; however, none were patients being treated with dabigatran. Of the 15 patients on warfarin, 3 of them underwent surgeries associated with their head injuries. All 3 surgeries were a right craniotomy. Of the 25 patients who were not receiving anticoagulants, 4 of them underwent surgeries associated with their head injuries: 1 right craniotomy, 1 right frontotemporal craniotomy, 1 bilateral craniotomy, and 1 left frontotemporal parietal craniotomy.

Associated anticoagulant/antiplatelet aggregates in the dabigatran group were warfarin in 2 of 5 , aspirin in 2 of 5 , and clopidogrel in 1 of 5 , while the warfarin group included aspirin in 3 of 15 and clopidogrel in 3 of 15 . The mean total number of comorbidities was 4.4 in the dabigatran group, 4.2 in the warfarin group, and 2.6 in the NAC group. The most common comorbidities in the dabigatran group were atrial fibrillation in 4 of 5 , coronary artery disease in 1 of 5 , hypertension in 2 of 5 , cardiomyopathy in 2 of 5 , and hypercholesterolemia in 1 of 5 . The most common comorbidities in the warfarin group were hypertension in 13 of 15 , atrial fibrillation in 12 of 15 , hypercholesterolemia in 5 of 15 , coronary artery disease in 5 of 15 , and dementia in 5 of 15 . The most common comorbidities in the NAC group were hypertension in 15 of 25 , hypercholesterolemia in 10 of 25 , dementia in 4 of 25 , and depression in 4 of 25 .

All of the following mean times for the dabigatran group are from time of arrival to our facility. Four of 5 patients received rFVIIa, with a mean transfusion start time of 118.5 minutes (range 25-132 minutes). Four of 5 patients received transfusions of FFP, 3 of 5 patients received platelets, and 2 of 5 patients received FFP and platelets. Two of 5 patients received dialysis, with a mean start time of 298 minutes (range 60-566 minutes) (Fig. 1). The mean dialysis time was 441 minutes (range 255-627 minutes). All patients had repeat CT scans during reversal of anticoagulation, of which 4 of 5 obtained in the dabigatran group showed new or expanded hemorrhages, while the warfarin group had 3 of 15 ( $p=0.03$, Fisher exact test). Of those patients undergoing repeat $\mathrm{CT}$ scans in the NAC group, 4 of 25 showed progression. The radiologist on call, who remained blinded to both the patient's clinical findings and anticoagulant medication, interpreted the results. The mean ICU length of stay was 4 days (range 2-6 days) in the dabigatran group, 3.2 days (range 1-10 days) in the warfarin group, and 2.3 days (range 0-13 days) in the NAC group.

The total number of CHIs from ground-level falls at our institution during 2010 was 376, with an overall mortality rate of $14 \%$. The overall mortality rate for patients sustaining CHI was $2(40 \%)$ of 5 in the dabigatran group, $0(0 \%)$ of 15 in the warfarin group, and $0(0 \%)$ of 25 in the NAC group (Table 1). The 2 deaths of the patients on dabigatran were related to the progression of the bleeding/hemorrhage, which cascaded to multiorgan failure and ultimately death. 


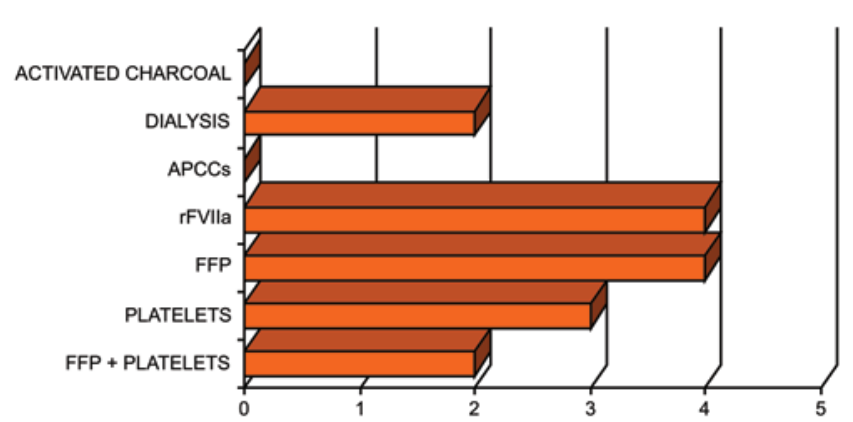

Fig. 1. Graph showing dabigatran reversal decisions (numbers on $x$ axis denote the number of patients with anticoagulation reversal). APCCs $=$ activated PCCs.

\section{Discussion}

Dabigatran etexilate is an oral prodrug that is rapidly converted by a serum esterase into a potent, direct, competitive inhibitor of thrombin. It is approved as an alternative to warfarin for risk reduction in stroke and systemic embolism in patients with nonvalvular atrial fibrillation. The kidneys excrete $80 \%$ of the standard given dose, and its serum half-life is $12-17$ hours. The time to peak drug concentration occurs approximately 1.5-3 hours after oral administration, with the maximal effect on coagulation being within 2 hours..$^{14}$ Due to its predictable anticoagulation response dabigatran does not require routine anticoagulation monitoring, which can be seen as a major advantage over warfarin. Furthermore, dabigatran is not affected by changes in diet, and it has fewer drug-to-drug interactions compared with its counterpart warfarin. ${ }^{18}$ The Randomized Evaluation of Long-term Anticoagulation Therapy (RELY) trial demonstrated a reduction in the risk of stroke when comparing warfarin to dabigatran: from $1.57 \%$ (warfarin) to $1.01 \%$ (dabigatran). Intracranial bleeding was reduced from $0.74 \%$ (warfarin) to $0.30 \%$ (dabigatran), and life-threatening bleeding from $1.80 \%$ (warfarin) to $1.45 \%$ (dabigatran), without any significant change in overall major bleeding-3.36\% (warfarin) to $3.11 \%$ (dabigatran). Life-threatening bleeding was a subcategory of major bleeding that consisted of fatal bleeding, symptomatic intracranial bleeding, bleeding with a decrease in the hemoglobin level of at least $50 \mathrm{~g} / \mathrm{L}$, or bleeding requiring transfusion of at least $4 \mathrm{U}$ of blood or inotropic agents, or necessitating surgery. Major bleeding was defined as a reduction in the hemoglobin level of at least $20 \mathrm{~g} / \mathrm{L}$, transfusion of at least $2 \mathrm{U}$ of blood, or symptomatic bleeding in a critical area or organ. ${ }^{2}$ Total mortality rates were also lowered from $4.13 \%$ per year on warfarin to $3.64 \%$ for patients on dabigatran..$^{21}$ All these benefits make dabigatran an attractive alternative to its anticoagulant predecessor.

Unintentional falls are the leading cause of injuryrelated deaths and emergency department visits in the elderly population in the US. ${ }^{1}$ As the population ages, more injuries from falls will be seen in the near future. A recent report found that falls from standing account for $27.3 \%$ of trauma admissions and are the second greatest consumer of hospital and ICU days. For patients 65 years of age and older, falls accounted for $43 \%$ of all injuries. ${ }^{1}$ Mem-

TABLE 1: Comparison between study groups in 45 patients with $\mathrm{ICH}^{*}$

\begin{tabular}{|c|c|c|c|c|c|}
\hline Characteristic & NAC Group & Dabigatran Group & Warfarin Group & Statistics & $\begin{array}{l}\text { Dabigatran vs Warfarin } \\
\text { ( } p \text { values) }\end{array}$ \\
\hline no. of pts (total) & 25 & 5 & 15 & & \\
\hline $\operatorname{sex}$ & & & & FET & 1 \\
\hline male & 12 & 3 & 10 & & \\
\hline female & 13 & 2 & 5 & & \\
\hline mean age in yrs & 75 & 81.6 & 83.9 & $t=0.751$ & 0.46 \\
\hline mean ISS & 13.6 & 20 & 16.47 & $t=-0.876$ & 0.39 \\
\hline \multicolumn{6}{|l|}{ mean GCS score } \\
\hline at presentation & 14.2 & 14.6 & 14.6 & $t=0.000$ & 1 \\
\hline at discharge & 14.8 & $14.3 \dagger$ & 14.5 & $t=0.256$ & 0.80 \\
\hline \multicolumn{6}{|l|}{ CT findings } \\
\hline SAH & 11 & 4 & 3 & & \\
\hline $\mathrm{SDH}$ & 12 & 1 & 12 & & \\
\hline IPH & 3 & 2 & 5 & & \\
\hline progression & 4 & 4 & 3 & FET & $0.03 \ddagger$ \\
\hline \multicolumn{6}{|l|}{ associated meds } \\
\hline warfarin & 0 & 2 & NA & & \\
\hline aspirin & 0 & 2 & 3 & FET & 0.56 \\
\hline clopidogrel & 0 & 1 & 3 & FET & 1 \\
\hline deaths & 0 & $2(40 \%)$ & 0 & FET & 0.05 \\
\hline
\end{tabular}


bers of the elderly population also have a worse prognosis compared with the younger population, demonstrated by a mortality rate of $24 \%$ after suffering a traumatic brain injury, compared with $12.8 \%$ in the nonelderly population. ${ }^{16}$ The elderly also experience greater morbidity, as illustrated by longer hospital stays, worse functional outcomes, lower discharge GCS scores, and lower functional independence measure scores compared with their younger counterparts. ${ }^{9,10}$ To complicate this matter even further, the prevalence of atrial fibrillation is strongly dependent on age, indicating that the use of oral anticoagulation therapy will probably substantially increase due to the projected aging of the US population.,

When comparing warfarin to dabigatran, the health care provider must remember that warfarin's anticoagulant effects can be rapidly reversed. Options include vitamin K, FFP, cryoprecipitate, PCCs (a preparation of concentrated forms of clotting factors II, VII, IX, and $\mathrm{X}$ that is used specifically to replace the factors that are inactivated by warfarin), factor IX complex, and rFVIIa (Novoseven). ${ }^{7,11,17}$ The best approach to warfarin reversal is controversial, and there are few published studies to guide best practice. The general consensus is that major or life-threatening bleeding requires rapid and complete warfarin reversal. ${ }^{4,8}$ The efficacy coupled with the nearuniversal availability of FFP in most countries results in FFP becoming a dominant tool for urgent warfarin reversal. Ivascu et al. ${ }^{6}$ found that the implementation of an aggressive reversal protocol was found to reduce time in triage (31 minutes vs 14 minutes), and the time to head CT scan from more than 2 hours to approximately 40 minutes. This reduced the time to initiation of reversal from more than 4 hours to less than 2 hours. More importantly, the death rate was decreased from $50 \%$ to $10 \%$ after implementing an aggressive warfarin protocol.

The combination of dabigatran's increasing popularity and our nation's aging baby boomer society leads to a potential dilemma. A specific agent and/or protocol for reversing the effects of dabigatran is currently unavailable in published clinical data. ${ }^{20}$ Our review of the current literature and preclinical studies suggests that future management options may include the use of activated charcoal, hemodialysis, and administration of rFVIIa and/or PCC..$^{12,20}$ On the basis of the mean concentration differences, the mean fraction of dabigatran removed by dialysis was $62 \%$ at 2 hours and $68 \%$ at 4 hours ${ }^{15}$ In their own pharmaceutical insert, the manufacturer (Boehringer Ingelheim Pharmaceuticals) suggests the use of FFP and/or packed red blood cells. However, although this may be a part of the usual treatment for bleeding, there is no clinical experience or evidence supporting the use of plasma products as a means to reverse the anticoagulant effect of dabigatran. ${ }^{20}$ Findings in healthy volunteers and ex vivo data suggest that rFVIIa antagonizes the anticoagulant effects of dabigatran by initiating homeostasis at sites of bleeding by directly activating thrombin on the surface of platelets in the absence of tissue factor. ${ }^{19,20} \mathrm{As}$ a result, this agent may have a potential off-label use for reversing anticoagulation in patients on dabigatran who sustain ICHs. ${ }^{12}$ Another consideration is activated PCCs (Fieba VH), which have shown significant reductions in bleeding times in ex vivo models, and complete reversal of dabigatran-inhibited thrombin potential by using human plasma in an in vitro study. ${ }^{13,19}$

To our knowledge, this is the first study to bring to light this dilemma. In our case series of 5 patients presenting with CHIs while on dabigatran, the patients had no significant difference in demographic or clinical characteristics with regard to sex, age, ISS, GCS scores, comorbidities, associated medications, mechanism of injury, or type of ICH. Despite these similarities between both groups, our analyses showed a statistically significant difference in terms of injury progression seen on CT scans $(p=0.03$, Fisher exact test) and a clinically significant higher mortality rate in the dabigatran group $(\mathrm{p}=$ 0.05 , Fisher exact test; Table 1). ${ }^{6}$

As already discussed, the benefits and application of an anticoagulation reversal protocol is key to improving treatment and outcome in the case of warfarin. The lack of an established protocol and a specific antidote led to a substantial variance at our institution in the treatment of the patients on dabigatran. None of the 5 patients received activated charcoal. Only 4 of the 5 patients received rFVIIa, with a mean transfusion start time of 118.5 minutes (range 35-132 minutes). Four of 5 patients received transfusions of FFP, 3 of 5 received platelets, and 2 of 5 received FFP and platelets (Fig. 1). Only 2 of 5 patients received dialysis, with a mean start time of 441 minutes (range 255-627 minutes) after presentation. All 5 patients had repeat CT scans during reversal, 4 of which showed new or expanding hemorrhages. This demonstrates dabigatran's devastating potential predicament. On review of our treatment approach, a great deal of variance was observed in regard to the time and type of treatment received. Because of these data, and the literature cited earlier, our institution is establishing a reversal protocol for the treatment of patients on dabigatran presenting with $\mathrm{CHI}$ (Fig. 2).

\section{Conclusions}

Classic anticoagulants, such as warfarin, have reasonable efficacy and specific antidotes, but also have undesirable features. Newer anticoagulants such as dabigatran were developed to overcome these undesirable features, but in return do not have a specific reversal protocol. As the use of dabigatran becomes more ubiquitous in the population with atrial fibrillation, it is critical for physicians involved in the care of patients with $\mathrm{CHI}$ to be aware of an elevated mortality rate in these patients if no treatment protocol or guideline is in place. The intention of this paper is not simply to bring to light the higher mortality rate associated with $\mathrm{CHI}$ in patients on dabigatran, but to the fact that physicians are not prepared to deal with it. In an attempt to improve efficacy and safety in our treatment approach, we will soon implement a reversal management protocol for patients with $\mathrm{CHI}$ on dabigatran at our institution in a prospective study (Fig. 2). This study was limited by the small population size, its retrospective design, having no standardized method to evaluate the hemorrhage blood volume, and the fact that patients were on multiple other anticoagulants, potentially altering their outcomes. 


\section{DABIGATRAN REVERSAL GUIDELINE FOR CLOSED HEAD INJURY}

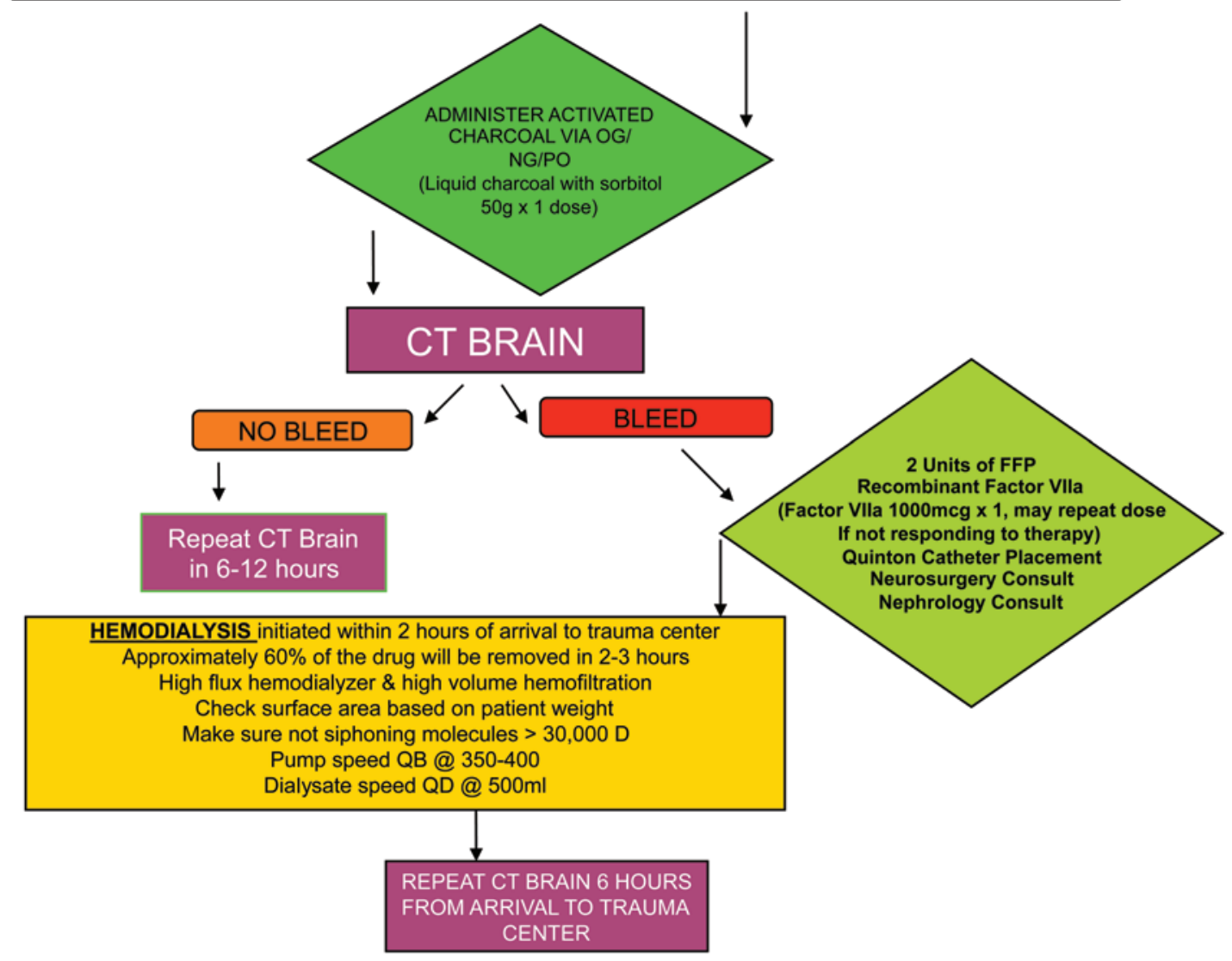

FIG. 2. Chart showing dabigatran reversal protocol for patients with $\mathrm{CHI}$.

\section{Disclosure}

The authors report no conflict of interest concerning the materials or methods used in this study or the findings specified in this paper.

Author contributions to the study and manuscript preparation include the following. Conception and design: Parra. Acquisition of data: Parra, Johnson, Gullett, Wichner, Kokaram. Analysis and interpretation of data: Parra, Johnson, Avila, Wichner. Drafting the article: Parra, Johnson. Critically revising the article: all authors. Reviewed submitted version of manuscript: all authors. Approved the final version of the manuscript on behalf of all authors: Parra. Statistical analysis: Parra, Johnson, Avila. Administrative/technical/ material support: Parra, Gullett, Wichner, Kokaram. Study supervision: Parra.

\section{References}

1. American College of Surgeons: National Trauma Databank Report 2006, Version 6.0. (http://www.facs.org/trauma/ntdb/ pdf/ntdbannualreport2006.pdf) [Accessed March 5, 2013]

2. Connolly SJ, Ezekowitz MD, Yusuf S, Eikelboom J, Oldgren J, Parekh A, et al: Dabigatran versus warfarin in patients with atrial fibrillation. N Engl J Med 361:1139-1151, 2009

3. Go AS, Hylek EM, Phillips KA, Chang Y, Henault LE, Selby JV, et al: Prevalence of diagnosed atrial fibrillation in adults: national implications for rhythm management and stroke prevention: the AnTicoagulation and Risk Factors in Atrial Fibrillation (ATRIA) Study. JAMA 285:2370-2375, 2001

4. Hanley JP: Warfarin reversal. J Clin Pathol 57:1132-1139, 2004
5. Howard JL II, Cipolle MD, Horvat SA, Sabella VM, Reed JF III, Fulda G, et al: Preinjury warfarin worsens outcome in elderly patients who fall from standing. J Trauma 66:1518-1524, 2009

6. Ivascu FA, Howells GA, Junn FS, Bair HA, Bendick PJ, Janczyk RJ: Rapid warfarin reversal in anticoagulated patients with traumatic intracranial hemorrhage reduces hemorrhage progression and mortality. J Trauma 59:1131-1139, 2005

7. Kalina M, Tinkoff G, Gbadebo A, Veneri P, Fulda G: A protocol for the rapid normalization of INR in trauma patients with intracranial hemorrhage on prescribed warfarin therapy. Am Surg 74:858-861, 2008

8. Makris M, Watson HG: The management of coumarin-induced over-anticoagulation annotation. Br J Haematol 114: 271-280, 2001

9. Osler T, Hales K, Baack B, Bear K, Hsi K, Pathak D, et al: Trauma in the elderly. Am J Surg 156:537-543, 1988

10. Perdue PW, Watts DD, Kaufmann CR, Trask AL: Differences in mortality between elderly and younger adult trauma patients: geriatric status increases risk of delayed death. J Trauma 45:805-810, 1998

11. Safaoui MN, Aazami R, Hotz H, Wilson MT, Margulies DR: A promising new alternative for the rapid reversal of warfarin coagulopathy in traumatic intracranial hemorrhage. Am J Surg 197:785-790, 2009

12. Sartori MT, Imbergamo S, Zanon E, Bonaccorso G, Pittoni G, Feltracco P, et al: Effect of recombinant activated factor VII in critical bleeding: clinical experience of a single center. Clin Appl Thromb Hemost 15:628-635, 2009

13. Sørensen B, Ingerslev J: A direct thrombin inhibitor studied 


\section{Dabigatran bleed risk with closed head injuries}

by dynamic whole blood clot formation. Haemostatic response to ex-vivo addition of recombinant factor VIIa or activated prothrombin complex concentrate. Thromb Haemost 96:446453, 2006

14. Stangier J: Clinical pharmacokinetics and pharmacodynamics of the oral direct thrombin inhibitor dabigatran etexilate. Clin Pharmacokinet 47:285-295, 2008

15. Stangier J, Rathgen K, Stähle H, Mazur D: Influence of renal impairment on the pharmacokinetics and pharmacodynamics of oral dabigatran etexilate: an open-label, parallel-group, single-centre study. Clin Pharmacokinet 49:259-268, 2010

16. Susman M, DiRusso SM, Sullivan T, Risucci D, Nealon P, Cuff S, et al: Traumatic brain injury in the elderly: increased mortality and worse functional outcome at discharge despite lower injury severity. J Trauma 53:219-224, 2002

17. Testerman GM, Shilad S, George KJ: Rapid warfarin reversal with factor VIIa in an elderly trauma patient with retroperitoneal hematoma. Tenn Med 102:37-39, 2009

18. Tran A, Cheng-Lai A: Dabigatran etexilate: the first oral anticoagulant available in the United States since warfarin. Cardiol Rev 19:154-161, 2011

19. van Ryn J, Ruehl D, Priepke H, Hauel N, Wienen W: Reversibility of the anticoagulant effect of high doses of the direct thrombin inhibitor dabigatran, by recombinant Factor VIIa or activated prothrombin complex concentrate. Haematologica 93 (Suppl 1):148, 2008 (Abstract)

20. van Ryn J, Stangier J, Haertter S, Liesenfeld KH, Wienen W, Feuring M, et al: Dabigatran etexilate-a novel, reversible, oral direct thrombin inhibitor: interpretation of coagulation assays and reversal of anticoagulant activity. Thromb Haemost 103:1116-1127, 2010

21. Wallentin L, Yusuf S, Ezekowitz MD, Alings M, Flather M, Franzosi MG, et al: Efficacy and safety of dabigatran compared with warfarin at different levels of international normalised ratio control for stroke prevention in atrial fibrillation: an analysis of the RE-LY trial. Lancet 376:975-983, 2010

Manuscript submitted March 28, 2012.

Accepted March 4, 2013.

Please include this information when citing this paper: published online May 1, 2013; DOI: 10.3171/2013.3.JNS12503.

Address correspondence to: Michael W. Parra, M.D., Department of Surgery, Nova Southeastern University School of Medicine, Delray Medical Center/Provisional Level I Trauma Center, 5352 Linton Boulevard, Delray Beach, Florida 33484. email: michaelwparra@ yahoo.com. 